Check for updates

Cite this: RSC Adv., 2019, 9, 2474

\title{
Sakura-based activated carbon preparation and its performance in supercapacitor applications
}

\begin{abstract}
Fei Ma, (D) *a Shaolan Ding, ${ }^{a}$ Huijun Ren ${ }^{\mathrm{b}}$ and Yanhua Liu ${ }^{\mathrm{a}}$
3D porous carbonaceous materials were prepared by combining pre-carbonization and $\mathrm{KOH}$ activation with sakura petals as raw materials. The prepared porous sakura carbon (SAC-4) exhibits a high specific surface area, a suitable pore size distribution, a low proportion of oxygen-rich groups and $\mathrm{N}$ functional groups, and a partially graphitized phase, which are very beneficial for the electrochemical performance of the material as a supercapacitor electrode. In the activation step, when the mass ratio of $\mathrm{KOH}$ to sakura carbon (SC) is 4, a supercapacitor is prepared. A maximal specific capacitance of $265.8 \mathrm{~F} \mathrm{~g}^{-1}$ is obtained when the current density is $0.2 \mathrm{~A} \mathrm{~g}^{-1}$. When the current density is $1 \mathrm{~A} \mathrm{~g}^{-1}$, after 2000 cycles in succession, the capacitance retention rate is excellent and the cycling stability can reach as high as $90.2 \%$. The obtained results indicate that porous carbon prepared with sakura blossom as the raw material is an effective and environmentally friendly electrode material for energy storage.
\end{abstract}

Received 25th November 2018 Accepted 28th December 2018

DOI: 10.1039/c8ra09685f

rsc.li/rsc-advances

which satisfies the conditions of different functional pores with different sizes required in supercapacitor applications. Among the different types of pores, mesopores/macropores can provide ion transport channels, which can significantly improve the rate performance, while micropores can contribute to a large specific surface area to help accumulate additional charges and obtain a high specific capacitance. ${ }^{11}$ The design of layered porous structures can be achieved by many approaches, such as physical activation ${ }^{12}$ or chemical activation, ${ }^{13}$ polymer blending carbonization, ${ }^{14}$ and the use of soft templates ${ }^{15}$ and hard templates associated with carbonization or activation. ${ }^{16,17}$ Chemical activation is an efficient method with a low cost that can be used to develop porous structures that can be prepared at lower temperatures within a shorter activation time. At present, biomass raw materials including soybean, nori, cabbage, candlenut flower, loofah, potatoes, egg shell, silkworms, and peanut shells have been used to synthesize bio-carbon and applied for the electrode materials of supercapacitors. ${ }^{18-26}$ Biomass carbon has exhibited good effects in supercapacitor applications, but the porous carbon in sakura petals has rarely been investigated.

Sakura is a woody plant of the Cerasus genus in the Rosaceae family, mainly distributed in temperate regions, especially in China, Japan and South Korea. Most sakura trees in China are used as garden ornamental plants, and the withered petals can only be burned or discarded, which causes a large number of sakura petals to be wasted and creates some pressure on the environment. The petals are flat in form and have a thickness of only $10-20 \mu \mathrm{m}$, and there are some fine folds forming channels that supply water on the petal surface. ${ }^{27}$ These characteristics make sakura petals advantageous as

${ }^{a}$ College of Bioresources Chemical and Materials Engineering, Shaanxi University of Science \& Technology, Xi'an 710021, China.E-mail: mf3060303201@yeah.net

${ }^{b}$ School of Arts and Sciences of Shaanxi University of Science \& Technology, Xi'an 710021, China 

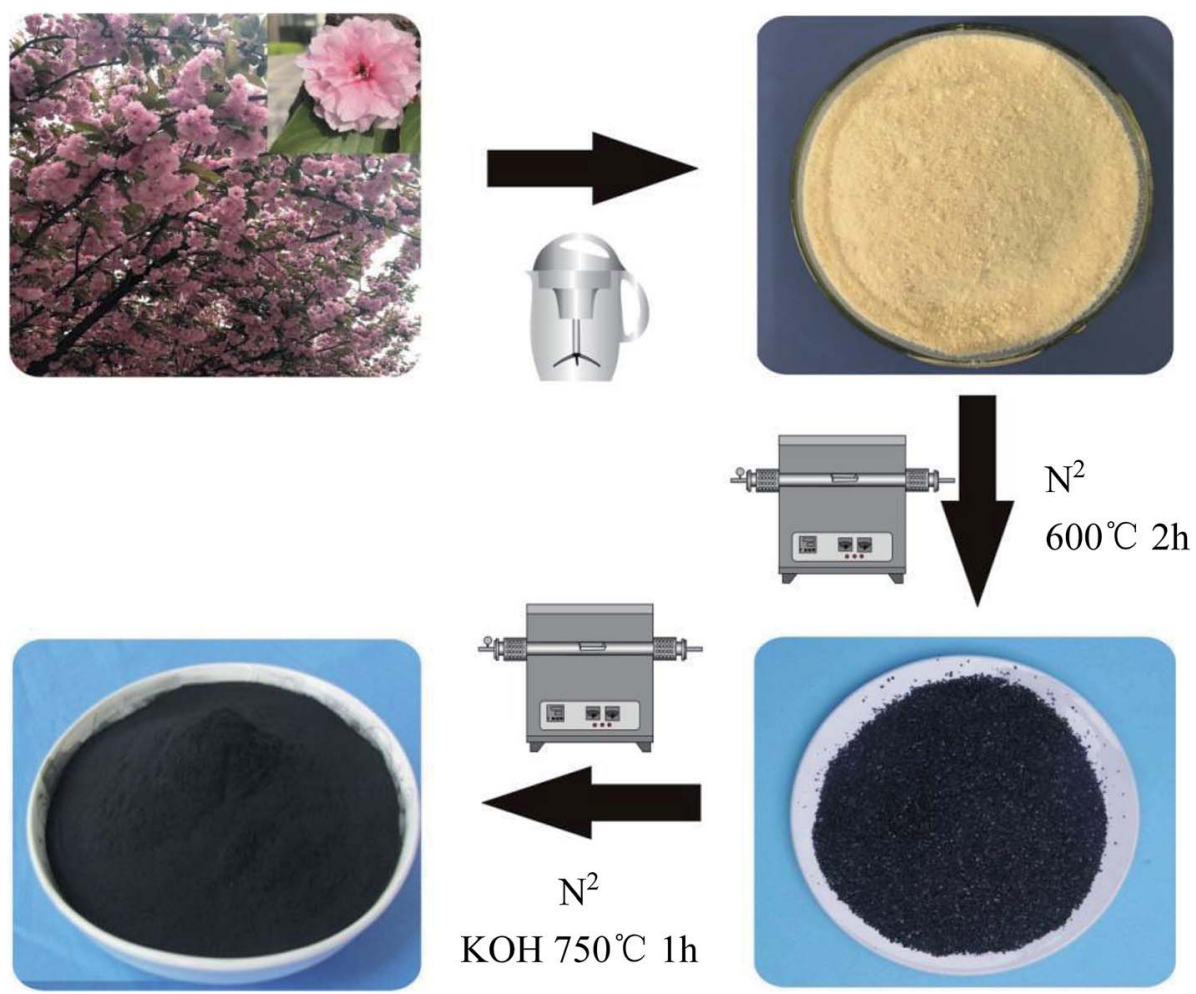

Fig. 1 Schematic diagram of the preparation of the sakura-based porous carbon.

precursors for preparing porous carbon with a higher specific surface area.

In this study, sakura-based activated carbon with a porous structure was prepared by the methods of collection, drying, carbonization, $\mathrm{KOH}$ chemical activation, and acid/water washing. Electrodes were then fabricated and the electrochemical performance of the sakura-based activated carbon was tested by cyclic voltammetry (CV), constant current charge/ discharge (GCD) and electrochemical impedance spectroscopy (EIS). The results show that the prepared sakura-based activated carbon exhibits a high specific surface area and a high specific capacitance. The maximum specific capacitance of the sakurabased activated carbon is $265.8 \mathrm{~F} \mathrm{~g}^{-1}$ when the current is $0.2 \mathrm{~A} \mathrm{~g}^{-1}$ in a three-electrode system. In addition, the sakurabased activated carbon electrodes have better cycling stability. These properties may make sakura-based activated carbon become a novel source of carbonaceous materials for highperformance supercapacitors.

\section{Experimental methods}

\subsection{Materials and reagents}

The sakura petals used to prepare the activated carbon in this study were collected on the campus of Shaanxi University of Science \& Technology, China. Potassium hydroxide $(\mathrm{KOH})$ was purchased from Titan Technology Co., Ltd. (Shanghai, China). Polytetrafluoroethylene (PTFE) was purchased from Lizhiyuan Co., Ltd. (Taiyuan, China). $N$-Methylpyrrolidone (NMP) was purchased from Lizhiyuan Co., Ltd. (Taiyuan, China).
Acetylene black was purchased from Tianyi Co., Ltd. (Tianjin, China). Nickel foam was purchased from Honor Technology Co., Ltd. (Shanghai, China). All the chemical reagents were of analytical grade and could be used without any further purification.

\subsection{Preparation of the sakura-based activated carbon}

A brief description of the process for preparing the sakurabased activated carbon is shown in Fig. 1. The exact process is as follows. The flower stalks were removed from the collected sakura. The petals were crushed and then ultrasonically washed with absolute ethyl alcohol and deionized water for 10 minutes, respectively. After being dried in an oven at $60{ }^{\circ} \mathrm{C}$, the powder was placed in a corundum ark and put into a tube furnace. In a nitrogen atmosphere, the temperature was increased to $600{ }^{\circ} \mathrm{C}$ for $2 \mathrm{~h}$, at a heating rate of $5{ }^{\circ} \mathrm{C} \mathrm{min}{ }^{-1}$, and then it was naturally cooled to room temperature. The carbonized sakura powder and a potassium hydroxide solution were mixed and the powder was impregnated for $24 \mathrm{~h}$ at the mass ratios of $1: 3,1: 4$ and $1: 5$, dried in an oven at $80{ }^{\circ} \mathrm{C}$, placed in a corundum ark and put into a tube furnace. The temperature was increased to $750{ }^{\circ} \mathrm{C}$ to activate the materials for $1 \mathrm{~h}$, with a heating rate of $5{ }^{\circ} \mathrm{C} \min ^{-1}$, and the temperature was then naturally cooled to room temperature. Then, they were washed with 2 mol of hydrochloric acid, deionized water and absolute ethyl alcohol, dried in an oven at $80{ }^{\circ} \mathrm{C}$, and ground into a powder in an agate mortar, which completed the preparation of the samples of the sakura-based activated carbon. The obtained sample was named as SAC- $x(x$ 
stands for the impregnation ratio of $\mathrm{KOH}$, where $x=3,4$, or 5 ). For comparison, carbonized sakura powder was incubated for $1 \mathrm{~h}$ without adding $\mathrm{KOH}$ at $750{ }^{\circ} \mathrm{C}$, and then the obtained sample was named as SC.

\subsection{Preparation of electrode plates}

The sample, the adhesive (PTFE) and the conductive agent (acetylene black) were weighed according to the mass ratio of $8: 1: 1$ and the solvent of NMP in moderation was added to the mixture to be ground for $20 \mathrm{~min}$. After the slurry was prepared, it was uniformly coated onto a foamed nickel current collector treated with acetone, absolute ethanol and deionized water, and dried (the area of the foamed nickel sheet was $1 \mathrm{~cm} \times 2 \mathrm{~cm}$, in which the coated area was only $1 \mathrm{~cm} \times 1 \mathrm{~cm}$ ). After the coating was finished, it was placed in an oven and dried at $60^{\circ} \mathrm{C}$ for $12 \mathrm{~h}$. After being taken out, it was tablet-formed at $10 \mathrm{MPa}$ on a tableting machine to complete the preparation of the electrode sheets (the mass of the active material was about $2.5 \mathrm{mg}$ ).

\subsection{Material characterization instruments}

The morphology of the sample was observed using an S-4800 field emission scanning electron microscope (FE-SEM), made in Hitachi, Japan, with an accelerating voltage of $15 \mathrm{kV}$. A JEM$2100 \mathrm{~F}$ high resolution transmission electron microscope (HRTEM) was used to characterize the microstructure of the product. A Nicolet 6700 Fourier transform infrared spectrometer was used to characterize the surface functional groups of the sample. The phases of the obtained sample were analyzed by an X-ray powder diffractometer of Type X-pert, whose wavelength was $\lambda=0.15416 \mathrm{~nm}$, and the scanning angle was 10 to 80 degrees. Utilizing an ASAP-2460 automatic adsorption instrument manufactured by Micromeritics Company, USA, a liquid nitrogen adsorption-desorption experiment was performed on the samples at a liquid nitrogen temperature of 77 K. The specific surface area was calculated by the multi-point Brunauer-Emmett-Teller (BET) method and the pore size distribution was calculated according to Barrett-JoynerHalenda (BJH) theory. The sample ingredients were analyzed by a PHI-1600 X-ray photoelectron spectrometer manufactured by PE Company, America.

\subsection{Electrochemical characterization}

The electrochemical characterization test was done in a threeelectrode system. The measurements of electrochemical impedance spectroscopy (EIS), cyclic voltammetry (CV) and constant current charge-discharge (GCD) were performed on a CHI 660E electrochemical workstation. The foamed nickel sheets coated with the electrode material were used as the working electrodes, a $2 \mathrm{~cm} \times 2 \mathrm{~cm}$ platinum sheet electrode was used as a counter electrode, and a saturated calomel electrode (SCE) was used as a reference electrode. The reaction electrolyte was a $6 \mathrm{~mol} \mathrm{~L}^{-1} \mathrm{KOH}$ solution. The EIS frequency range was from $100 \mathrm{kHz}$ to $10 \mathrm{mHz}$ at open circuit voltage. The alternating current amplitude was $5 \mathrm{mV}$, and the electrochemical window of the $\mathrm{CV}$ test was from -1 to $0 \mathrm{~V}$. The scanning rates were $10,20,50,100$, and $200 \mathrm{mV} \mathrm{s}^{-1}$, respectively.

\section{Results and discussion}

\subsection{Morphology and microstructure}

The morphology and the microstructure of the samples were characterized by SEM. As shown in Fig. 2, Fig. 2a is the activated sakura carbon without adding $\mathrm{KOH}$, which has a wrinkled and rough morphology on the surface. This is mainly due to the surface shrinkage caused by the evaporation of water, volatile substances and decomposed gaseous substances during the high temperature carbonization as shown in Fig. 2a. As the mass ratio of $\mathrm{KOH}$ to the pre-carbonized precursor is increased from 3 to 5 , it can be seen in Fig. 2 b$\mathrm{d}$ that the vascular-like stripes gradually disappear, replaced by a porous and rougher surface. Apparently, during alkali etching, there are hollowing effects that result in structures similar to 3D honeycombs. These structures are composed of interconnected macropores and carbon walls, ${ }^{28}$ which facilitates the rapid transmission of ions and optimizes the rate performance of the supercapacitors. ${ }^{29}$ At the same time, these large pores are open, providing a large number of interfaces for charge storage. When the $\mathrm{KOH}$ mass ratio is 5 , the pore structure collapses, which is disadvantageous for the storage of charge.

The TEM images in Fig. 3a-e reveal these changes more clearly, further demonstrating the porous structure obtained by activation treatment under high temperatures. By comparison, it can be seen that the alkali activation step produces more micropores, mesopores and even macropores, but excessive $\mathrm{KOH}$ can cause the pores to collapse. When the $\mathrm{KOH}$ ratio is only 4, higher specific surface areas and porosity are observed. This is advantageous as such specific surface areas and porosity promote the charge accumulation in the electric double layer capacitor.

In addition, the structure of the sakura carbon is further studied using XRD patterns. In Fig. 4 a, two broad peaks appear between $22^{\circ}$ and $43.9^{\circ}$ for all of the SAC- $x$ materials, which are similar to the (002) and (100) characteristic peaks of graphite. ${ }^{30}$ Furthermore, it is clear that the peaks become wide and their intensities are decreased as $x$ is increased, especially for the (002) diffraction peak. The results show that the $\mathrm{KOH}$ etching reduces the regularity of the SC structure due to the formation of pores or defects, which resembles the reported literature. ${ }^{31}$

The structure of the partially graphitized carbon was further confirmed by Raman spectroscopy. ${ }^{32-35}$ As shown in Fig. 4b, for all the samples, the two broad peaks at 1304 and $1590 \mathrm{~cm}^{-1}$ are the D band and G band, respectively. The D band reflects the defects and disorder of the carbon material, while the $\mathrm{G}$ band is related to the bond extension of the $\mathrm{sp}^{2}$ carbon atom pair. ${ }^{36,37}$ The degree of graphitization is reflected by the intensity ratio of $I_{\mathrm{D}} / I_{\mathrm{G}}$, and a lower ratio indicates a higher degree of graphitization. $\mathrm{KOH}$ activation can produce nanopores and defects in the carbon framework. Therefore, an increase in the amount of $\mathrm{KOH}$ should result in a lower degree of graphitization. In fact, the activated sample (SAC- $x$ ) shows a larger $I_{\mathrm{D}} / I_{\mathrm{G}}$ value than SC. In addition, for SAC-3, SAC-4 and 

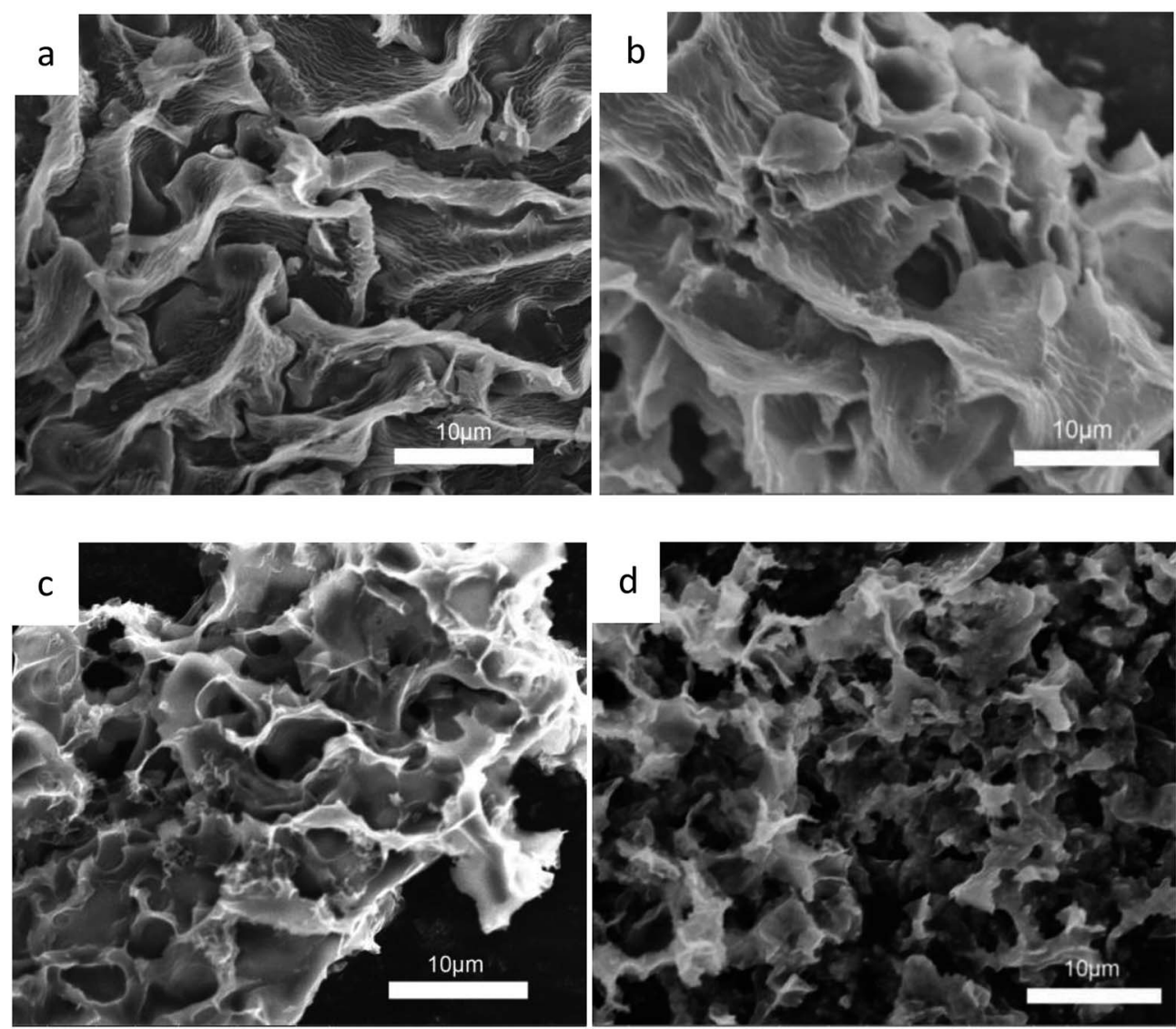

Fig. 2 SEM images of SC (a), SAC-3 (b), SAC-4 (c) and SAC-5 (d) at the same magnification.

SAC-5, the $I_{\mathrm{D}} / I_{\mathrm{G}}$ values are increased from 0.97 for SC to 0.99 , 1.02 and 1.05, respectively. The increased defects can be used as active and adsorption sites so as to improve the performance and catalytic activity of the supercapacitors.

The surface chemical compositions of SC and SAC- $x$ were analyzed by XPS, as shown in Fig. 5 a. Both SC and SAC- $x$ show three peaks with binding energies of 285, 400 and $532 \mathrm{eV}$, which are characteristic of the C1s, N1s and O1s orbits, indicating that there are $\mathrm{C}, \mathrm{N}$ and $\mathrm{O}$ elements present in the samples. The content of $\mathrm{C}, \mathrm{N}$ and $\mathrm{O}$ elements in the SC and SAC- $x$ samples is shown in Table 1 . With an increase in $\mathrm{KOH}$ ratio, the atomic content of $\mathrm{N}$ is decreased from $0.69 \%$ to $0.33 \%$, and the content of $\mathrm{O}$ is decreased from $30.2 \%$ to $9.41 \%$. The fine analyses of the C1s and N1s spectra in the SC and SAC-4 samples by means of the peak differentiating fitting method are shown in Fig. 5b. The corresponding electron binding energy and the corresponding functional groups in the $\mathrm{C} 1 \mathrm{~s}$ spectrum are $\mathrm{C}=\mathrm{C}$ (284.6 eV), C-N (285.5 eV), C-OH $(286.5 \mathrm{eV})$ and $\mathrm{O}-\mathrm{C}=\mathrm{O}(289.3$ $\mathrm{eV}){ }^{38}$ respectively. The $\mathrm{N} 1 \mathrm{~s}$ spectrum shows that pyridine-Noxygen (402.7 eV), pyrrole/pyridone- $\mathrm{N}(400.2 \mathrm{eV})$ and pyridine nitrogen $(398.3 \mathrm{eV})$ coexist in SAC-4. ${ }^{11}$ The presence of the oxygen-rich groups and the $\mathrm{N}$-functional groups in a low proportion will provide the necessary hydrophilicity and additional capacitance for the supercapacitor. The oxygen functional groups in the aqueous electrolytes mainly occur in the following reactions: $:^{39-42}$

$$
\begin{gathered}
\mathrm{C}-\mathrm{OH} \Leftrightarrow \mathrm{C}=\mathrm{O}+\mathrm{H}^{+}+\mathrm{e}^{-} \\
-\mathrm{COOH} \Leftrightarrow \mathrm{COO}+\mathrm{H}^{+}+\mathrm{e}^{-} \\
\succ \mathrm{C}=\mathrm{O}+\mathrm{e}^{-} \Leftrightarrow \succ \mathrm{C}-\mathrm{O}-
\end{gathered}
$$

where reactions (1) and (2) only indicate quasi-reversible or irreversible properties in alkaline and neutral media, and the faradaic pseudo-capacitance mainly comes from reaction (3). It is worth noting that oxygen functional groups can also improve the wettability between the electrode material and the electrolyte. In addition, the nitrogen functional groups with additional free electrons not only help to improve wettability, but also introduce faradaic pseudo-capacitance in the aqueous electrolyte ${ }^{39,42}$ which is favorable for SAC-4 to be a supercapacitor electrode material. ${ }^{43}$

$\mathrm{N}_{2}$ adsorption-desorption analysis was performed so as to study the porosity of the prepared materials. According to the classification by the International Union of Pure and Applied Chemistry (IUPAC), all of the SAC- $x$ materials in Fig. 6a have a higher $\mathrm{N}_{2}$ adsorption capacity and show Type-I adsorption isotherm characteristics. When the relative pressure is $P / P_{0}<$ 0.05 , the volumetric adsorption is sharply increased, which indicates that there exist plenty of micropores. ${ }^{\mathbf{1 0}}$ For the samples, accompanying the Type-H4 hysteresis loop $\left(p / p_{0}=\right.$ 0.45), a typical Type-IV isotherm can be observed, indicating 

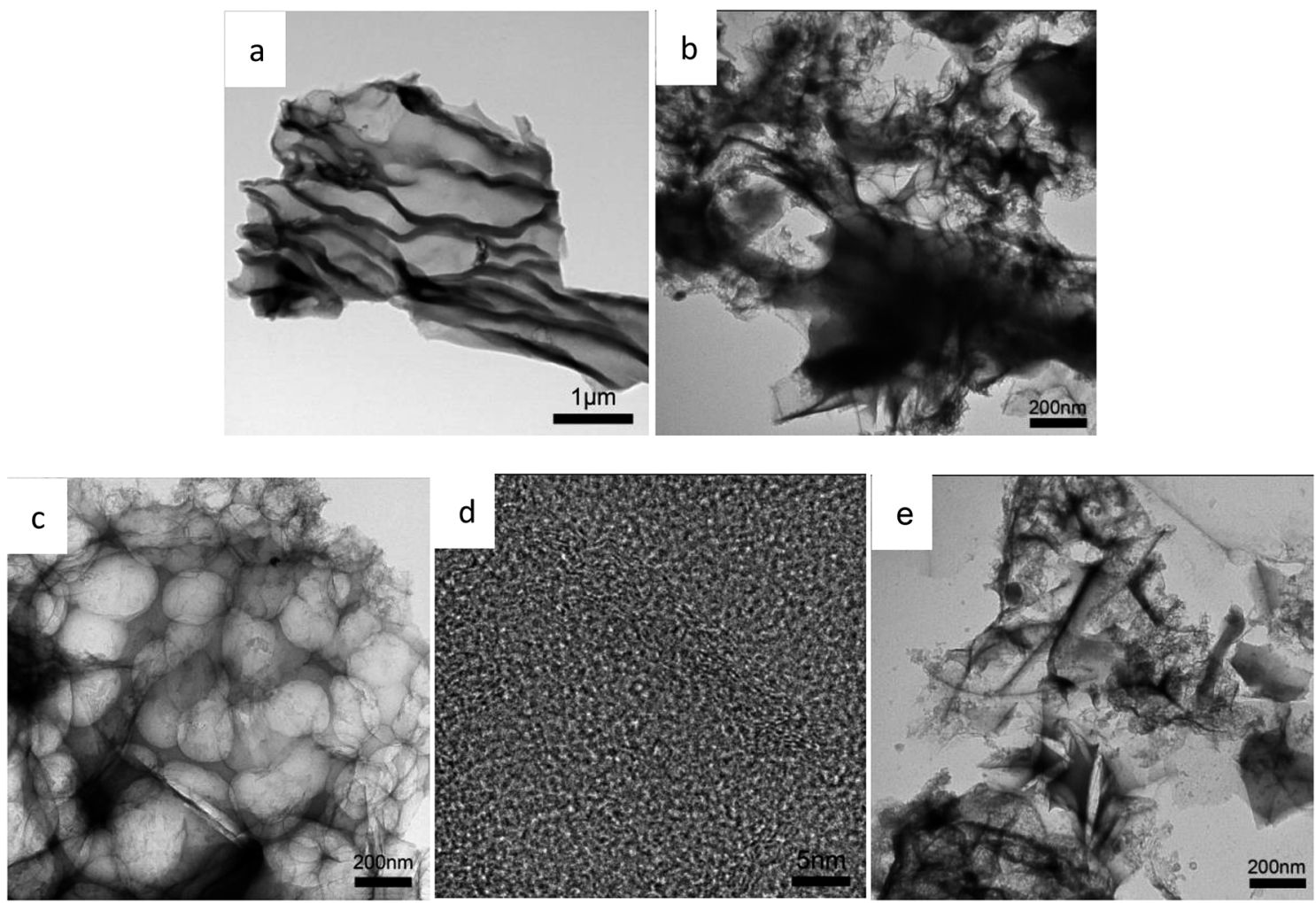

Fig. 3 TEM images of SC (a), SAC-3 (b), SAC-4 (c and d) and SAC-5 (e) at different magnifications.

that there exist micropores and mesopores. ${ }^{44}$ When the relative pressure is close to 1 , the adsorption capacity is slightly increased, showing that the samples also contain a small quantity of macropores. ${ }^{45}$

The pore characteristics of all the samples are summarized in Table 2. When the dose of $\mathrm{KOH}$ is increased from $x=3$ to 5 , the specific surface areas of SAC-3, SAC- 4 and SAC-5 are increased from 1137.70 to 1433.76 to $1785.41 \mathrm{~m}^{2} \mathrm{~g}^{-1}$, respectively. From SAC-3 to SAC-4, the external surface areas are almost unchanged, and the micropore areas are increased from 817.33 to $1099.55 \mathrm{~m}^{2} \mathrm{~g}^{-1}$. When the KOH dose is increased to 5 , the mesopore surface area is increased from 334.21 to 778.29 $\mathrm{m}^{2} \mathrm{~g}^{-1}$. The micropore area is slightly reduced (from 1099.55 to $\left.1007.12 \mathrm{~m}^{2} \mathrm{~g}^{-1}\right)$. This trend indicates that the primary micropores are produced mainly by inserting $\mathrm{KOH}$ or its corresponding metal $\mathrm{K}$ into the carbon layer and etching the carbon skeleton. ${ }^{46}$ With higher alkali doses, the corrosive materials not only produce more micropores, but also further etch the walls of the micropores, enlarge the pore size and reduce the proportion of microporous area, ${ }^{47}$ leading to such an irregular trend. It is obvious that when the optimal KOH dose is 4 , the micropore area is maximal. The pore size distributions of the SC and SAC- $x$ samples are obtained based on the theory of BJH. As shown in Fig. 6a, pores with a size of $0-2 \mathrm{~nm}$ are dominant among the porous structures of all the samples. This kind of microporosity can benefit charge storage to great extent, thereby enhancing the capacitance of the electrodes. ${ }^{48}$ Compared with the SAC- $x$ sample, the unactivated SC shows
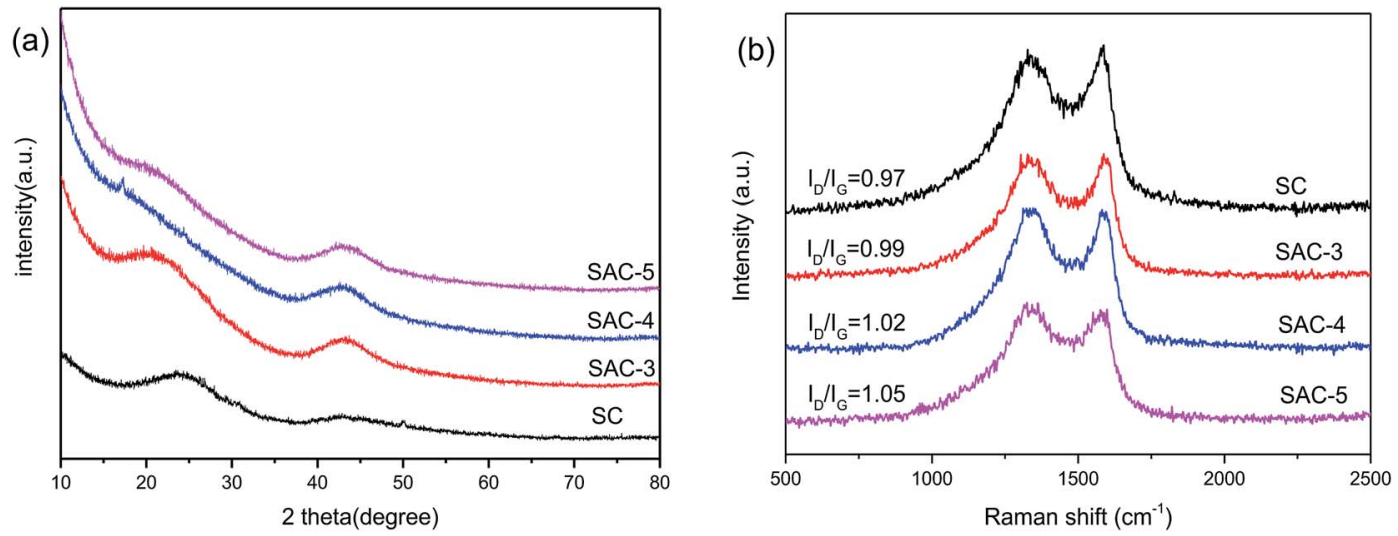

Fig. 4 (a) XRD patterns of SC and SAC- $x$, and (b) Raman images of SC and SAC-4. 

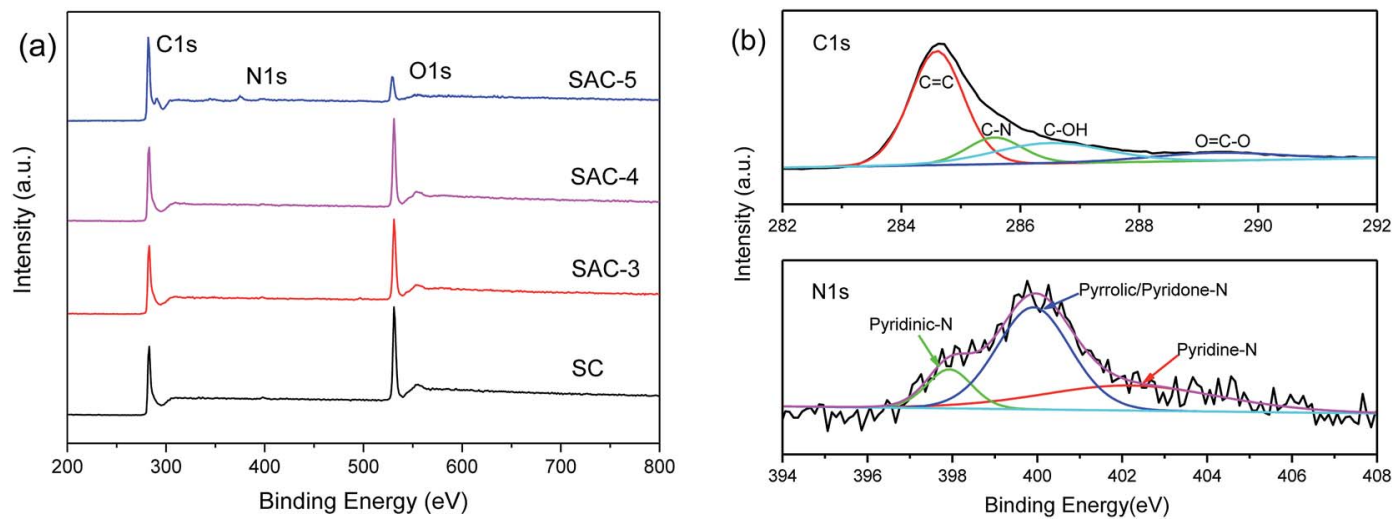

Fig. 5 (a) XPS spectra of SC and SAC-X, and (b) C1s and N1s peak-differentiating-fitting in the XPS spectra of SAC-4.

Table 1 Surface element composition determined by XPS

\begin{tabular}{llll}
\hline Sample & C (\%) & O (\%) & N (\%) \\
\hline SC & 69.11 & 30.2 & 0.69 \\
SAC-3 & 72.38 & 27.19 & 0.43 \\
SAC-4 & 72.92 & 26.58 & 0.5 \\
SAC-5 & 90.26 & 9.41 & 0.33
\end{tabular}

a much smaller BET surface area $\left(267.04 \mathrm{~m}^{2} \mathrm{~g}^{-1}\right)$ and a relatively smaller porosity.

\subsection{Electrochemical performance}

Due to the higher BET specific surface area and different types of pore structures, the electrochemical performance of SAC- $x$ as a supercapacitor electrode is expected. In order to study the capacitance performance of sakura-based activated carbon, CV and constant current charge/discharge measurements were performed in the potential window of -1.0 to $0 \mathrm{~V}$. Fig. 7 a shows the $\mathrm{CV}$ curves of the electrode with a scan rate of $100 \mathrm{mV} \mathrm{s}^{-1}$. The curves present approximately rectangular shapes, showing double-layer capacitance behavior. As mentioned above, due to the presence of oxygen functional groups and a small amount of $\mathrm{N}$ element, the redox reaction causes a high voltage hump from $-0.6 \mathrm{~V}$ to $-0.2 \mathrm{~V}$, which is similar to the reported literature. ${ }^{11}$ Moreover, it is clear that the $\mathrm{CV}$ curve area of SAC-4 is
Table 2 Specific surface areas and pore volumes of SC and SAC-x

\begin{tabular}{|c|c|c|c|c|c|c|c|}
\hline \multirow[b]{2}{*}{ Sample } & \multicolumn{3}{|c|}{ BET SSA $\left(\mathrm{m}^{2} \mathrm{~g}^{-1}\right)$} & \multicolumn{3}{|c|}{$\begin{array}{l}\text { Pore volume } \\
\left(\mathrm{cm}^{3} \mathrm{~g}^{-1}\right)\end{array}$} & \multirow{2}{*}{$\begin{array}{l}\text { Average pore } \\
\text { width (nm) }\end{array}$} \\
\hline & Total & Micro & Meso & Total & Micro & Meso & \\
\hline SC & 267.04 & 202.16 & 64.88 & 0.15 & 0.10 & 0.05 & 4.7 \\
\hline SAC-3 & 1137.70 & 817.33 & 320.37 & 0.69 & 0.41 & 0.28 & 4.6 \\
\hline SAC-4 & 1433.76 & 1099.55 & 334.21 & 0.78 & 0.55 & 0.23 & 3.9 \\
\hline SAC-5 & 1785.41 & 1007.12 & 778.29 & 0.84 & 0.51 & 0.33 & 3.3 \\
\hline
\end{tabular}

larger than those of the SC and other SAC- $x$ samples, which means that SAC- 4 has the highest capacitance. The CV curves (10-200 $\mathrm{mV} \mathrm{s}^{-1}$ ) of SAC-4 at different scanning rates are shown in Fig. $7 \mathrm{~b}$ and also maintain approximate rectangular shapes at $200 \mathrm{mV} \mathrm{s}^{-1}$. This is mainly ascribed to the SAC-4 pore structure and the larger micropore area, as well as the relatively short diffusion distance of the dielectric ions from the mesopores to the micropores. ${ }^{49}$

The GCD curves of SC and SAC- $x$ when the current density is $1 \mathrm{~A} \mathrm{~g}^{-1}$ are shown in Fig. 7c. The curves show isosceles triangle shapes, indicating the better capacitive behavior of the EDLC. The slight distortion may be caused by the pseudo-capacitance generated by the functional groups containing heteroatoms $(\mathrm{N}$ and $\mathrm{O}$ ). The specific capacitance of the GCD curves can be calculated using eqn (4):
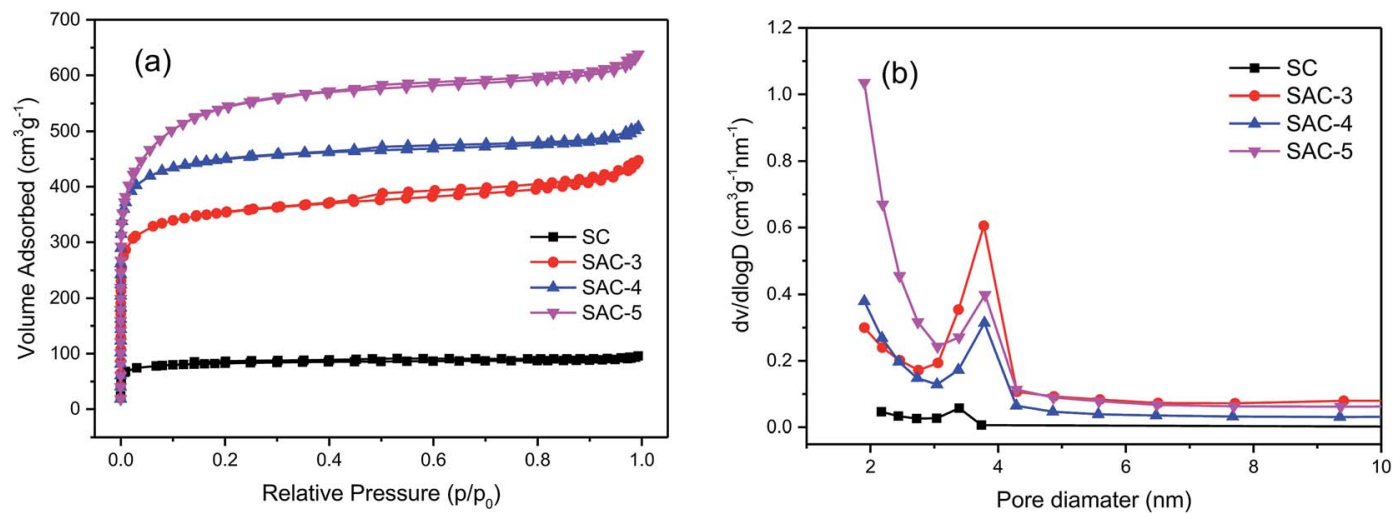

Fig. 6 (a) $\mathrm{N}_{2}$ adsorption isotherms of SC and SAC- $x$, and (b) pore size distributions of SC and SAC- $x$. 

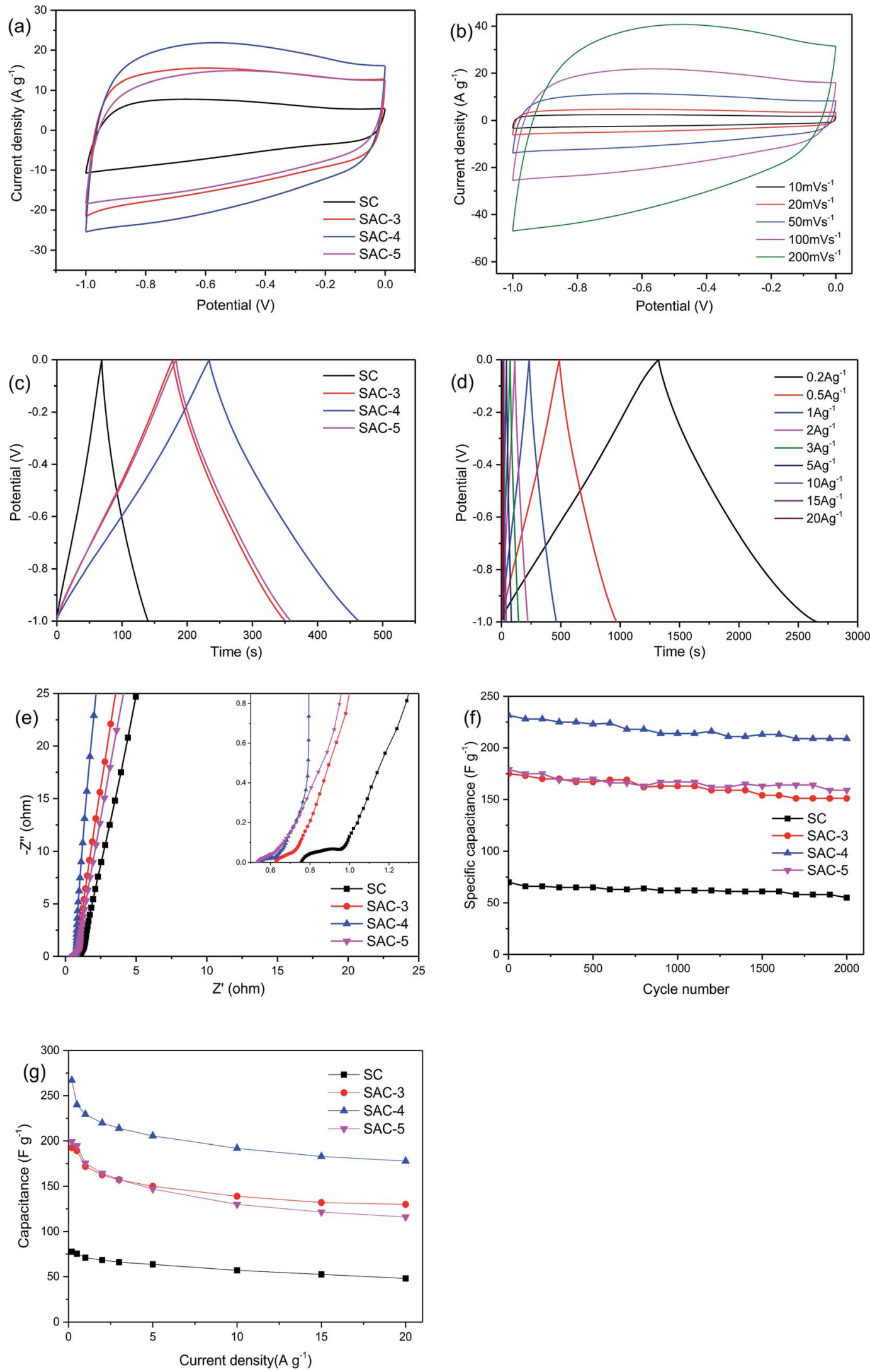

Fig. 7 Electrochemical performance of SC and SAC-x in a three-electrode system: (a) CV curves of SC and SAC-x at $100 \mathrm{mV} \mathrm{s}^{-1}$; (b) CV curves of SAC-4 at different scan rates; (c) GCD curves of SC and SAC-x at $1 \mathrm{~A} \mathrm{~g} \mathrm{~g}^{-1}$; (d) GCD curves of SAC-4 at different current densities; (e) Nyquist diagrams of SC and SAC- $x$; (f) cycle life tests of SC and SAC-x at $1 \mathrm{~A} \mathrm{~g}^{-1}$; (g) specific capacitances of SC and SAC at 0.2-20 A g ${ }^{-1}$. 
Table 3 Specific capacitances of various waste biomass-based activated carbons

\begin{tabular}{|c|c|c|c|c|c|}
\hline Material & $S_{\text {BET }}\left(\mathrm{m}^{2} \mathrm{~g}^{-1}\right)$ & $\begin{array}{l}\text { Specific capacitance } \\
\left(\mathrm{F} \mathrm{g}^{-1}\right)\end{array}$ & $\begin{array}{l}\text { Current density } \\
\left(\mathrm{Ag}^{-1}\right)\end{array}$ & Electrolyte & Ref. \\
\hline Soybean & 1749 & 243.2 & 0.5 & $6 \mathrm{M} \mathrm{KOH}$ & 18 \\
\hline Perilla frutescens & 655 & 270 & 0.5 & $6 \mathrm{M} \mathrm{KOH}$ & 20 \\
\hline Paulownia flower & 1471 & 297 & 1 & $1 \mathrm{M} \mathrm{H}_{2} \mathrm{SO}_{4}$ & 21 \\
\hline Loofah & 2718 & 309.6 & 1 & $6 \mathrm{M} \mathrm{KOH}$ & 22 \\
\hline Silkworm & 2523 & 304 & 1 & $6 \mathrm{M} \mathrm{KOH}$ & 25 \\
\hline Peanut shell & 1565 & 245 & 1 & $6 \mathrm{M} \mathrm{KOH}$ & 26 \\
\hline Sakura & 1433.8 & 265.8 & 0.2 & $6 \mathrm{M} \mathrm{KOH}$ & This work \\
\hline
\end{tabular}

$$
C=\frac{I \Delta t}{m \Delta V}
$$

where $I / m$ is the current density $\left(\mathrm{A} \mathrm{g}^{-1}\right), \Delta t$ is the discharge time (s), and $\Delta V$ is the potential range $(\mathrm{V})$. The specific capacitances of SC, SAC-3, SAC-4, and SAC-5 are calculated to be 69.9, 175, 231.6, and $179 \mathrm{~F} \mathrm{~g}^{-1}$, respectively when the current density is $1 \mathrm{~A} \mathrm{~g}^{-1}$. Clearly, it can be seen that the specific capacitance of SAC- $x$ is much higher than that of SC because their porous structures are activated by $\mathrm{KOH}$, which enhances the kinetics of ion and electron transport at the electrode/electrolyte interface..$^{50}$ It is worth noting that SAC-4 shows a much longer discharge time than SC and SAC- $x$ ( 3 and 5), which means that it has the largest specific capacitance. As mentioned above, the larger specific capacitance may be due to the bigger specific surface area and the bigger total pore volume. The GCD curves of the SAC-4 electrode at different current densities are shown in Fig. 7d. The approximate symmetry and the linear GCD curves confirm the reversible charge-discharge process, consistent with the $\mathrm{CV}$ results mentioned above, which is a characteristic of an ideal capacitor. The specific capacitance is decreased with an increase of the current density, which may be attributed to the slow diffusion and the migration of protons through the electrodes. ${ }^{51}$ When the current density is $0.2 \mathrm{~A} \mathrm{~g}^{-1}$, the maximal specific capacitance of SAC- 4 is $265.8 \mathrm{~F} \mathrm{~g}^{-1}$. As listed in Table 3, SAC-4 exhibits a specific capacitance that is almost equal to or even higher than that of other biomassderived carbon materials reported in the literature.

The electrochemical impedance spectra of the electrode material are shown in Fig. 7e. As is well known, the Nyquist diagram mainly consists of three parts: a semicircle in the high frequency area, a straight line with a slope of $45^{\circ}$ in the low frequency area, and a straight line with a slope of more than $45^{\circ}$ in the extreme low frequency area. As shown in the partial enlargement in the inset of Fig. 7e, the intercept of the real axis represents the series resistance $\left(R_{\mathrm{s}}\right)$, which is the sum of the ionic resistance of the electrolyte, the resistance of the intrinsic active material, and the contact resistance between the electrode and the collector electrode. ${ }^{52,53}$ For the SC, SAC-3, SAC-4 and SAC-5 capacitors, the $R_{\mathrm{S}}$ values are $0.77,0.62,0.55$ and $0.47 \mathrm{~V}$, respectively. At the same time, SAC- 4 exhibits a semicircle with a smaller diameter than the other semicircles. This semicircle is related to the Faraday process of the charge transfer at the electrode/electrolyte interface. A smaller semicircle means that the charge transfer is faster and the resistance is smaller. In addition, the straight line with a slope close to 1 for SAC-4 also indicates that ions have a faster transfer rate on the electrode surface. ${ }^{54,55}$ The cycling stability is another key parameter that determines the practical application of supercapacitors. The cycle lives of the four electrodes of SC and SAC- $x$ were tested in $6 \mathrm{M} \mathrm{KOH}$ electrolyte when the current density was $1 \mathrm{~A} \mathrm{~g}^{-1}$. As shown in Fig. 7f, after 2000 consecutive chargedischarge cycles, the specific capacitance of the SAC- 4 electrode was maintained as $209 \mathrm{~F} \mathrm{~g}^{-1}$, which corresponds to $90.2 \%$ of its initial value, indicating that the SAC- 4 electrode has excellent cycling stability.

The specific capacitance of sakura carbon was calculated as shown in Fig. $7 \mathrm{~g}$. Because SC is not activated, it exhibits poor performance. After activation with $\mathrm{KOH}$, the specific capacitance first increased and then decreased. It can be seen that excess KOH does not favor an increase of the specific capacitance. When the current density is $0.5 \mathrm{~A} \mathrm{~g} \mathrm{~g}^{-1}$, the specific capacitances of SAC-3, SAC- 4 and SAC- 5 are $189.4 \mathrm{~F} \mathrm{~g}^{-1}, 240 \mathrm{~F}$ $\mathrm{g}^{-1}$ and $194.8 \mathrm{~F} \mathrm{~g}^{-1}$, respectively. When the current density is increased to $20 \mathrm{~A} \mathrm{~g}^{-1}$, the initial specific capacitance retention rates of SAC-3, SAC- 4 and SAC- 5 are $68.6 \%, 74.1 \%$ and $59.5 \%$, respectively. Such changes may be related to the changes in the porous structure and the reduction in the degree of graphitization.

\section{Conclusions}

A carbonaceous material with a porous structure is prepared by combining simple carbonization and $\mathrm{KOH}$ activation with sakura petals as raw materials, and the porous properties can be controlled by changing the dose of $\mathrm{KOH}$. The functional groups on the surface of the prepared porous carbon were characterized using FT-IR spectroscopy, showing that functional groups containing more oxygen are introduced after the alkali activation. According to BET and BJH theories, the pore size distributions, the average pore diameters and the specific surface areas of the samples were calculated so as to determine that the specific surface area of SAC-4 is $1433.8 \mathrm{~m}^{2} \mathrm{~g}^{-1}$ and the average pore diameter is $3.9 \mathrm{~nm}$. The SEM and TEM analyses further confirm that more micropores, mesopores and even 
macropores are produced in the alkali activation step. In a water-based three-electrode system, the sakura-derived activated carbon showed a specific capacitance of $265.8 \mathrm{~F} \mathrm{~g}^{-1}$ when the current density was $0.2 \mathrm{~A} \mathrm{~g}^{-1}$. After 2000 cycles, the initial specific capacitance is maintained as $90.2 \%$. Therefore, it can be concluded that SAC-4 has great potential as an excellent electrode material for practical applications in the future.

\section{Conflicts of interest}

There are no conflicts to declare.

\section{References}

1 Y. Liang, Y. Li, H. Wang and H. Dai, J. Am. Chem. Soc., 2013, 135, 2013-2036.

2 A. Divyashree, A. B. A. M. Shoriya, S. Yallappa, K. Chaitra, N. Kathyayini and H. Gurumurthy, J. Energy Chem., 2016, 25, 880-887.

3 X. Han, M. Funk, F. Shen, Y. Chen, Y. Li, C. Campbell, J. Dai, X. Yang, J. Kim, Y. Liao, J. Connell, V. Barone, Z. Chen, Y. Lin and L. Hu, ACS Nano, 2014, 8, 8255-8265.

4 A. Ehsani, E. Kowsari, M. Dashti Najafi, R. Safari and H. Mohammad Shiri, J. Colloid Interface Sci., 2017, 503, 1016.

5 E. Frackowiak and F. Béguin, Carbon, 2001, 39, 937-950.

6 Y. Zhang, S. Yu, G. Lou, Y. Shen, H. Chen, Z. Shen, S. Zhao, J. Zhang, S. Chai and Q. Zou, J. Mater. Sci., 2017, 52, 1120111228.

7 D. Xie, X. H. Xia, W. J. Tang, Y. Zhong, Y. D. Wang, D. H. Wang, X. L. Wang and J. P. Tu, J. Mater. Chem. A, 2017, 16, 7578-7585.

8 X. X. Gu, C. J. Tong, S. Rehman, L. M. Liu, Y. L. Hou and S. Q. Zhang, ACS Appl. Mater. Interfaces, 2016, 25, 1599116001.

9 D. P. Qiu, N. N. Guo, A. Gao, L. Zheng, W. J. Xu, M. Li, F. Wang and R. Yang, Electrochim. Acta, 2019, 294, 398-405.

10 J. Niu, J. J. Liang, R. Shao, M. Y. Liu, M. L. Dou, Z. L. Li, Y. Q. Huang and F. Wang, Nano Energy, 2017, 41, 285-292.

11 X. H. Wang, H. T. Zhou, E. Sheridan, J. C. Walmsley, D. D. Ren and D. Chen, Energy Environ. Sci., 2016, 9, 232-239.

12 Z. Y. Sui, Q. H. Meng and J. T. Li, J. Mater. Chem. A, 2014, 2, 9891-9898.

13 B. J. Zhu, K. X. Li, J. J. Liu, H. Liu, C. G. Sun, C. E. Snape and Z. X. Guo, J. Mater. Chem. A, 2014, 2, 5490-5498.

14 A. H. Lu, H. J. Bongard, F. Schueth, A. Q. Wang, T. Zhang, W. P. Zhang, G. H. Wang, D. Qian, W. C. Li and G. P. Hao, J. Am. Chem. Soc., 2011, 29, 11378-11388.

15 J. Liu, N. P. Wickramaratne, S. Z. Qiao and M. Jaroniec, Nat. Mater., 2015, 14, 763-774.

16 S. J. Yang, M. Antonietti and N. Fechler, J. Am. Chem. Soc., 2015, 137, 8269-8273.

17 H. L. Fan and W. Z. Shen, ACS Sustainable Chem. Eng., 2016, 4, 1328-1337.

18 R. Karthika, Y. S. Houa, S. M. Chena, A. Elangovanb, M. Ganesanb and P. Muthukrishnanc, J. Ind. Eng. Chem., 2016, 37, 330-339.
19 C. Wang and T. Liu, J. Alloys Compd., 2017, 696, 42-50.

20 B. Liu, Y. Liu, H. Chen, M. Yang and H. Li, J. Power Sources, 2017, 341, 309-317.

21 J. Chang, Z. Gao, X. Wang, D. Wu, F. Xu, X. Wang, Y. Guo and K. Jiang, Electrochim. Acta, 2015, 157, 290-298.

22 X. L. Su, J. R. Chen, G. P. Zheng, J. H. Yang, X. X. Guan, P. Liu and X. C. Zheng, Appl. Surf. Sci., 2018, 436, 327-336.

23 G. Ma, Q. Yang, K. Sun, H. Peng, F. Ran, X. Zhao and Z. Lei, Bioresour. Technol., 2015, 197, 137-142.

24 Z. Li, L. Zhang, B. S. Amirkhiz, X. Tan, Z. Xu, H. Wang, B. C. Olsen, C. M. B. Holt and D. Mitlin, Adv. Energy Mater., 2012, 2, 431-437.

25 C. Gong, X. Wang, D. Ma, H. Chen, S. Zhang and Z. Liao, Electrochim. Acta, 2016, 220, 331-339.

26 X. He, P. Ling, J. Qiu, M. Yu, X. Zhang, C. Yu and M. Zheng, J. Power Sources, 2013, 240, 109-113.

27 X. Yu, Y. Wang, L. Li, H. Li and Y. Shang, Sci. Rep., 2017, 7, 45378.

28 M. Y. Song, Y. Y. Zhou, X. Ren, J. F. Wan, Y. Y. Du, G. Wu and F. W. Ma, J. Colloid Interface Sci., 2019, 535, 276-286.

29 S. J. Song, F. W. Ma, G. Wu, D. Ma, W. D. Geng and J. F. Wan, J. Mater. Chem. A, 2015, 3, 18154-18162.

30 Y. Teng, E. Liu, R. Ding, K. Liu, R. Liu, L. Wang, Z. Yang and H. Jiang, Electrochim. Acta, 2016, 194, 394-404.

31 K. Wang, N. Zhao, S. Lei, R. Yan, X. Tian, J. Wang, Y. Song, D. Xu, Q. Guo and L. Liu, Electrochim. Acta, 2015, 166, 1-11.

32 N. N. Guo, M. Li, Y. Wang, X. K. Sun, F. Wang and R. Yang, ACS Appl. Mater. Interfaces, 2016, 8, 33626-33634.

33 W. S. Chen, H. P. Yu, S. Y. Lee, T. Wei, J. Li and Z. J. Fan, Chem. Soc. Rev., 2018, 47, 2837-2872.

34 N. N. Guo, M. Li, X. K. Sun, F. Wang and R. Yang, Green Chem., 2017, 19, 2595-2602.

35 C. Chen, D. F. Yu, G. Y. Zhao, B. S. Du, W. Tang, L. Sun, Y. Sun, F. Besenbacher and M. Yu, Nano Energy, 2016, 27, 377-389.

36 W. Qian, F. Sun, Y. Xu, L. Qiu, C. Liu, S. Wang and F. Yan, Energy Environ. Sci., 2014, 7, 379-386.

37 B. Liu, Y. Liu, H. Chen, M. Yang and H. Li, J. Power Sources, 2017, 341, 309-317.

38 C. Peng, X. B. Yan, R. T. Wang, J. W. Lang, Y. J. Ou and Q. J. Xue, Electrochim. Acta, 2013, 87, 401-408.

39 S. P. Wang, J. N. Zhang, P. Shang, Y. Y. Li, Z. M. Chen and Q. Xu, Chem. Commun., 2014, 50, 12091-12094.

40 Y. J. Kim, Y. Abe and T. Yanagiura, Carbon, 2007, 45, 21162125.

41 S. T. Senthilkumar, B. Senthilkumar, S. Balaji, C. Sanjeeviraja and R. Kalai Selvan, Mater. Res. Bull., 2011, 46, 1320.

42 J. N. Zhang, X. L. Zhang, Y. C. Zhou, S. J. Guo, K. X. Wang, Z. Q. Liang and Q. Xu, ACS Sustainable Chem. Eng., 2014, 2, 1525-1533.

43 T. E. Rufford, D. Hulicova-Jurcakova, Z. Zhu and G. Q. Lu, Electrochem. Commun., 2008, 10, 1594-1597.

44 J. C. Groen, L. A. A. Peffer and J. Pérez-Ramírez, Microporous Mesoporous Mater., 2003, 60, 1-17.

45 Q. Wang, J. Yan, Y. Wang, T. Wei, M. Zhang, X. Jing and Z. Fan, Carbon, 2014, 67, 119-127. 
46 K. Konno, Y. Ohba, K. Onoe and T. Yamaguchiet, Carbon, 2008, 46, 721.

47 J. Chmiola, G. Yushin, Y. Gogotsi, C. Portet, P. Simon and P. L. Taberna, Science, 2006, 313, 1760-1763.

48 R. Wang, P. Wang, X. Yan, J. Lang, C. Peng and Q. Xue, ACS Appl. Mater. Interfaces, 2012, 4, 5800-5806.

49 D. Liu, W. Zhang, H. Lin, Y. Li, H. Lu and Y. Wang, J. Cleaner Prod., 2016, 112, 1190-1198.

50 A. K. Mondal, K. Kretschmer, Y. Zhao, H. Liu, H. Fan and G. Wang, Microporous Mesoporous Mater., 2017, 246, 72-80.
51 P. Cheng, S. Gao, P. Zang, X. Yang, Y. Bai, H. Xu, Z. Liu and Z. Lei, Carbon, 2015, 93, 315-324.

52 L. Sun, C. Tian, Y. Fu, Y. Yang, J. Yin, L. Wang and H. Fu, Chemistry, 2014, 20, 564-574.

53 D. Puthusseri, V. Aravindan, S. Madhavi and S. Ogale, Energy Environ. Sci., 2014, 7, 728-735.

54 L. Chen, T. Ji, L. Mu and J. Zhu, Carbon, 2017, 111, 839-848. 55 Y. Li, G. Wang, T. Wei, Z. Fan and P. Yan, Nano Energy, 2016, 19, 165-175. 